\title{
DYNECOM: Augmenting Empathy in VR with Dyadic Synchrony Neurofeedback
}

\author{
Simo Järvelä \\ Department of Information \\ and Service Management, \\ Aalto University \\ simo.jarvela2@aalto.fi \\ Janne Timonen \\ Helsinki Institute for
Information Technology, \\ University of Helsinki \\ janne.timonen@cs.helsinki.fi
}

\author{
Mikko Salminen \\ Gamification Group, \\ Tampere University; \\ Department of Information \\ and Service Management, \\ Aalto University \\ mikko.salminen@ tut.fi \\ Kristiina Mannermaa \\ Department of Information \\ and Service Management, \\ Aalto University \\ kristiina.mannermaa@aalto.fi
}

\author{
Antti Ruonala \\ Helsinki Institute for \\ Information Technology, \\ University of Helsinki \\ antti.ruonala@aalto.fi
}

\begin{abstract}
In a novel experimental setting, we augmented a variation of traditional compassion meditation with our custom built VR environment for multiple concurrent users. The system incorporates respiration and brainwave based biofeedback that enables responsiveness to the shared physiological states of the users. The presence of another user's avatar in the shared virtual space supported low level social interactions and provided active targets for evoked compassion. We enhanced interoception and the deep empathetic processes involved in compassion meditation with real time visualizations of breathing rates and the level of approach motivation assessed from EEG frontal asymmetry, and the dyadic synchrony of those signals between the two users. We found how the different biofeedback types increased both the amount of physiological synchrony between the users and their self-reported empathy, illustrating how dyadic synchrony biofeedback can expand the possibilities of biofeedback in affective computing and VR solutions for health and wellness.
\end{abstract}

\section{Introduction}

In the past few years the development of virtual reality environments and related technology has been quick, and they have also been utilized as tools for forwarding personal well-being, for example as a platform for mindfulness based meditation practice [1, $2,3]$. A strong presence inducing and disturbance free virtual environment $[4,5,6]$ seems an ideal solution for quick stress management exercises during the work day, where longer breaks and separate physical spaces would be impractical. In this paper we present how we have extended that basic idea in two ways: firstly, by incorporating respiration and brain wave based neurofeedback and visualizations to support meditation, and secondly, by enabling social interaction in a shared social virtual space where more than one user can meditate simultaneously. Additionally, we present how these two advances can be tied together by measuring and visualizing dyadic physiological synchrony between users, enabling a system responsive to the shared physiological states of the users. Augmenting a variation of traditional compassion meditation with our VR system, we examined in a strictly controlled 
laboratory experiment, how the different neurofeedback types affected both the amount of physiological synchrony between the users of the shared space and their self-reported empathy.

\section{Theoretical background}

\subsection{VR meditation and biofeedback}

In general, meditation exercises include various practices and traditions that are often "complex emotional and attentional regulatory strategies developed for various ends, including the cultivation of well-being and emotional balance" [7]. The positive effects of various meditation practices include, for example, the treatment for insomnia [8] and headache [9], and reduced blood serum cortisol levels and reduced systolic and diastolic pressure and pulse rate [10]. Long term meditation has also been shown to lead to changes in the attention related brain areas [11]. Mindfulness related compassion and loving-kindness meditation styles have been found to increase social connectedness, positive affect, and empathy $[12,13]$.

Biofeedback - using measured physiological signals as a feedback to the user through visualizations etc. - has been increasingly utilized in augmented meditation, e.g. $[14,15]$. The central idea is that the additional information provided by biofeedback increases interoceptive awareness [16, 17], that is, helps the user to become more aware of her own state while meditating, and that increased awareness supports meditating by making it easier or more efficient. Typically such systems utilize heart based measures, electrodermal activity, or occasionally brain wave measures, that could provide information regarding arousal and stress levels of the user [18].

With the popularization of VR devices and the current raise of interest in mindfulness and similar activities, the combination of VR and meditation feels like a natural coupling. Having a dedicated own clear space for meditation, especially during work hours in the office environment, is quite challenging, but a simple VR solution can provide that personal distraction-free moment necessary for mindfulness. There are already various prototypes of VR guided meditation and relaxation protocols. For example, in Relaxation Island [19] the user could walk in a soothing environment using a sea shell shaped joystick for navigation; initial results indicated that the use of the system had a relaxing effect, as intended.

Biofeedback functionality has been utilized in some VR applications already. For example, Virtual Meditative Walk [2] teaches chronic pain patients a mindfulness based stress reduction technique, utilizing sound effects, immersive VR environment, and biofeedback using galvanic skin response (GSR) to control the weather conditions in the environment. In Meditation chamber [1], in addition to the GSR, breathing rate and blood volume pulse were utilized to control features of the environment.

Also, the information from brain electrical activation, obtained by the electroencephalography (EEG), can be used in generating feedback. While many other biosignals have been utilized in biofeedback, a limited number of systems utilizing neurofeedback exist, e.g. [20, 21], the recent neuroadaptive VR environment RelaWorld being one of the few. RelaWorld was targeted for the training of attention skills and also for relaxation [22, 3]. The feedback, utilized as levitation effect and increased opaqueness or fog in the environment (a tranquil shore view), was adaptive to the user's EEG alpha $(8-13 \mathrm{~Hz})$ and theta (4-6 Hz) power band changes. In previous studies these power band activations have been related to relaxedness and focused attention, respectively. Initial results implied that the neurofeedback functionality increased, for example, the self-reported sense of presence and EEG theta band power.

\subsection{Supporting empathic interactions in shared VR meditation}

Like RelaWorld, DYNECOM incorporates EEG based neurofeedback but adds social dynamics to the environment by having multiple simultaneous users sharing the same VR space. This is a novel combination of features that opens up the possibility to augment primal social processes in VR. Meditation is primarily a solitary endeavour, however, the various forms of loving-kindness and compassion meditation, while typically exercised alone, can be seen as social as they strongly focus on empathic feelings towards others. A natural extension of the this is to have the target of the compassion meditation present. In the VR the presence of another user is represented with an avatar. Cognitively, many aspects of traditional compassion meditation seem to involve empathy processes $[12,13]$.

Empathy can be defined, in the context of experimental research, as the merging of the feelings with someone, when sympathy can be defined as an awareness of the other's feelings but not experiencing those same feelings [23]. The spontaneous mimicry of the empathized other may, via emotional contagion, build the subjective feeling of empathy and thus emotional resonance [24, 25]. Previous studies have related empathy to various positive outcomes, for 
example, to increased willingness to help [26].

Electroencephalography (EEG) is a relevant method for studying meditation, after all, different cognitive and conscious states are related to respective neurophysiological states. Previous studies have shown that the electrical activation of the brain can be studied as the powers of various frequency bands, that are related to, for example, cognitive and affective processes [27, 28, 29]. The frontal asymmetry (FA) of the EEG measurements, quantified as a relative difference between activations of the anterior left and right regions, is one of the most widely used biomarker in empathy research. The frontal left and right regions are parts of two separate neural systems that are related to approach and withdrawal motivations, respectively [28, 29]. Interestingly, experienced empathy and also the ability to empathize are related to increased left frontal activation [30, 31, 32].

Physiological synchrony refers to the extent to which physiological signals of two or more people are associated with each other, such as a mutual increase in heart rate (HR) during a shared experience. Such indices can be calculated from the psychophysiological measurement data when they have been recorded from two or more persons simultaneously. While the idea of dyadic synchrony is not a new one, it has not been thoroughly studied and all the psychological phenomena related to it remain unmapped. In previous studies, the synchronization of psychophysiological activities between two persons has been related to empathy. For example, synchronization of the electrodermal activations (EDA, sweating of the hands) between a therapist and a patient was related to the patient's perception of the therapist's empathy towards him or her, in a study by Marci et al. [33]. In addition, matching physiological states has been related to empathic accuracy; more accurate ratings of a videotape were obtained when there was stronger physiological linkage between the rater and the person on the videotape [34].

Naturally, similarly as a single biosignal can be utilized as biofeedback in VR systems, so can dyadic synchrony indices. Dyadic neurofeedback, as utilized in DYNECOM, provides the users information on to what extent are their physiological signals in synchrony, and consequently, information on those social and empathy related processes that can be measured with physiological synchrony indices. This approach of implementing dyadic neurofeedback to a shared VR space is, to our knowledge, completely novel, and we conducted a controlled laboratory experiment to study its effects on empathy.

\section{System Overview}

\subsection{Hardware and setup}

The system hardware setup consists of four computers: two running the test and two for monitoring and recording. Biosignals are first recorded with Brain Products QuickAmp devices. The recording computer streams the physiological data to the network from BrainVision Recorder software in real time. Next OpenViBE [35] is run on the test computers to process the signals before sending them to the main program running through Unity3D [36], where visualizations, user-end recording and networking are handled. Virtual reality is provided through two Oculus Rift headsets with Oculus Touch hand controllers. 3D-models were made with Blender [37] and Adobe Photoshop [38] and some assets were acquired from Unity3D asset store.

\subsection{Design of the virtual environment}

DYNECOM is built around the metaphor of "sitting together by the campfire". This immersive setting shares several suitable aspects: It is a relaxed social situation around a shared activity, where nature provides a relaxing background, and the built-in elements balance the wilderness with familiarity. Each session starts with a minimalistic room for recording the participant's baseline neurophysiological activation. It is followed by the meditation environment consisting of six stone statues sitting in a ring on a small shrine-like platform. The platform is surrounded by a short wall and a forest background lit by a cloudy evening sky. Participants sit during the experiment and, in the virtual environment, possess one of the two statues connected by a bridge. From this perspective users see the bridge connecting to the opposite statue. To increase immersion, an audio track of pink noise resembling wind rustling through the trees was added to mask the background sound of the laboratory. Depending on the test condition, the bridge, the scene lights and the aura-like ring surrounding active statues show various visual effects or cues to inform the user of their current state. Scene layout is shown in Figure 1.

To further guide the participants' attention, a short wall was added to encircle the platform. It acts to discourage the tendency of the users to explore the scene and limits the field of view to the essential elements. From the perspective of immersion, as the wall blocks the view down the forest the space feels enclosed, safe and more intimate. Having an open view of the sky counters possible claustrophobic anxieties, as does the low height of the wall. This conveys the idea that if needed, one could easily leave from the situation. 


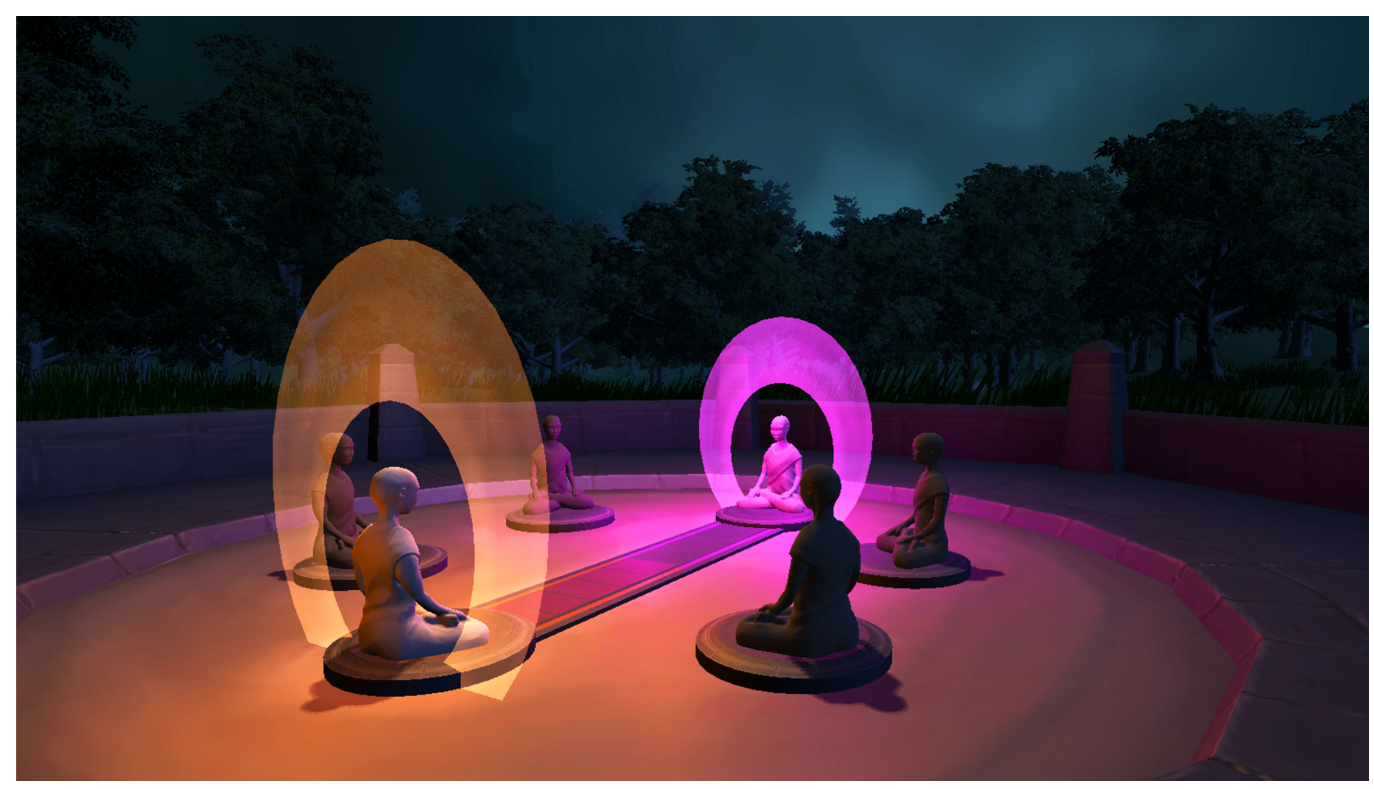

Figure 1. Overview of the scene elements and dyadic visualizations.

Compassion meditation often includes more than one target for the empathic feelings evoked during the exercise. Even though our focus is on dyadic interaction, this broader perspective was included in the design by having four passive statues sitting next to the two active ones connected by the bridge hinting at the possibility of a group setting.

As the study is not exploring the various effects elicited by the avatars themselves, the presentation of the participants must be extremely neutral while still being identifiable and easily approachable. Empathy towards virtual and artificial characters can be challenged by the so called uncanny valley effect, where imperfections in representation aiming for realism can cause a feeling of revulsion [39]. As a pragmatic solution, static statues are used as avatars as they offer a target for identification without expectations of expression.

To avoid biases and ease the identification, the statue is sculpted to be an androgynous and ageless humanoid lacking any strong identifiers. The face rests in a neutral relaxed expression and the statue sits legs crossed in a pose associated with meditative engagement in mental practice.

Virtual reality often causes discomfort commonly known as VR sickness. Symptoms include nausea, headache and disorientation after being exposed to virtual reality content. One potential cause for the symptoms is the disparity between perceived and real physical motion which causes conflict between sensory inputs [40]. This lead us to design a static scene, further supported with cues of embodiment in a statue. Dusk was chosen as scene lighting as a dark ambience acts as a contrasting background in the visual hierarchy, guiding the attention towards the cues and making them easily readable.

\subsection{Affective cues and neurofeedback}

The needs of the experimental research setting placed limitations for the visual design: The participants' two biosignals, frontal asymmetry variance and respiration, and their synchrony needed to be visually conveyed to both users through intuitive and non-distractive cues. Additionally, the activity must remain identical regardless of whether it is being conducted solo or in a dyadic condition.

The bridge connecting the two users acts as a display to convey biofeedback from both users. It lays at a convenient viewing angle and distance, enabling the users to perceive relevant states without needing to move their head [41]. A ring-like shape surrounds the active statues acting as an additional display for the state of the opposite participant.

Respiration is visualized with movement. The aura ring expands and contracts by the stages of breathing and a wave effect consisting of bars is shown on top of the bridge. The respiration bars consists of five consecutive layers that are enabled and illuminated with a fade in effect based on exhaling intervals. When an exhalation is detected the bar effect is launched by illuminating each of the layers one at a time, starting from the one closest to the user. After a short period of 


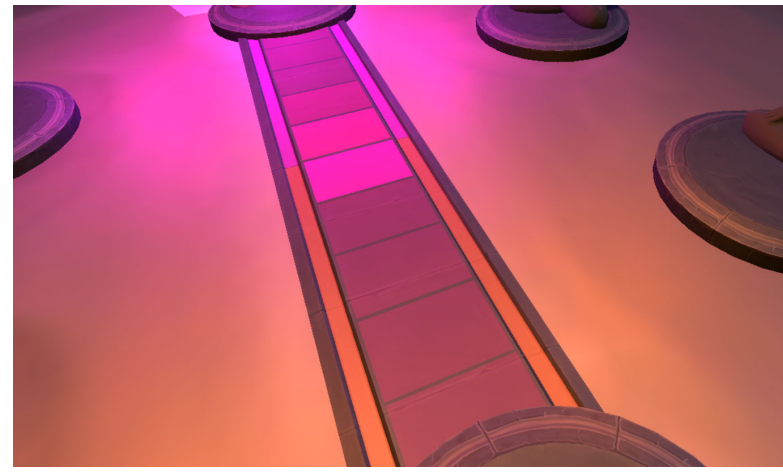

Figure 2. Breathing bar blink effect visualizing synchronous respiration

time the layers withdraw and fade out back to invisible if a new exhalation is not detected. In the dyadic condition when participants breathe at the same pace, the bars colliding in the middle of the bridge blink with a bright highlight indicating the synchrony of respiration. The respiration bars and synchronous blink effects are seen in Figure 2.

The idea behind the respiration bar visualization was to tie the effect closely to the sensor values so that the responsiveness of the effect seems instant and reminiscent to a frequency bar in graphic equalizers. When looking directly ahead, the lower part of the of the field of vision provides information supporting interoceptive awareness $[16,17]$, and most of the neutral orientation focuses on information about the state of the other.

In the environment different colors are used to represent the amount of empathy related approach motivation measured with the EEG. In other words, frontal asymmetry values are color encoded which acts as a visual cue for the current state of the user and their progress in meditation. Colors are present in all of the lights in the scene: the auras around the statues, the bridge side recesses, and in the breathing bars on the bridge. Colors vary in a gradient from cold green through yellow and orange to warm red and pink. Frontal asymmetry is calculated by a logarithmic formula presented by Allen et al. [42]. When the measured EEG states of both users reach the limit of synchrony the glowing effect visualizing this is activated. The glowing effect raises the intensity and brightness of the color and it appears on the recesses on both sides of the bridge. Lights, aura around a statue and the glowing bridge recesses are illustrated in Figure 3.

Algorithms calculating visualizations are designed to adapt to the participants throughout the subsessions. Individual minimum and maximum values of the monitored biosignals are tracked during the session and

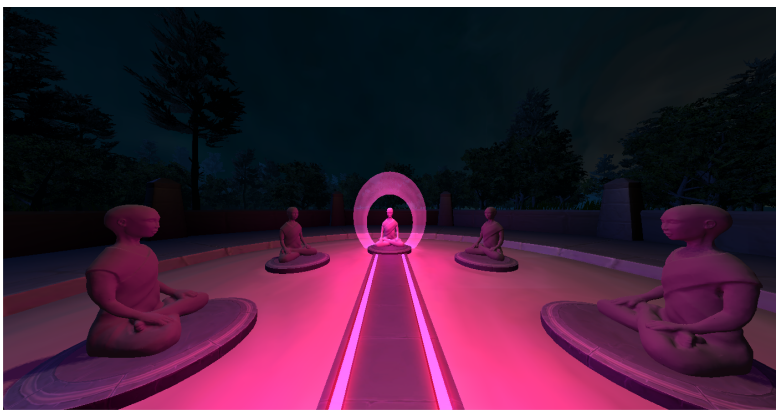

Figure 3. Glow cue in the bridge recesses for a synchronous EEG-state.

are used to define the individual's range. For frontal asymmetry, this range is then used in calculating the shown color value, using a moving average of the last 9 seconds of frontal asymmetry input. Reactivity of the algorithm was balanced through an iterative process scoping the parameters where participants felt that they could affect the visualization without it becoming too noisy. The adaptivity results in a system where users showing synchrony of color, meaning them being currently at the same respective percentage of their individual ranges at this moment in the session, even if the raw FA-values (frontal asymmetry) differ. Also, as the minimum and maximum values adapt to participant performance throughout the session, the same colors can represent different FA-values at the start and at the end of the session. Before the visualizations are engaged, a $30 \mathrm{~s}$ monitoring is used for defining a base range for the effects. OpenViBE epoch averaging and moving averages with the range calculations are used for fault tolerance.

\section{Experimental evaluation}

\subsection{Procedure}

Before the experiment, the participants filled in a background information form. The experiment was conducted on participant pairs, who took part in the experiment together. On their arrival, the participants gave informed consent to participate and they were given instructions regarding the operation of the virtual environment and what their task during the experiment would be. The participants were instructed to concentrate on empathic, warm and compassionate feelings and direct them at the statue representing their pair. They were also encouraged to utilize the information provided by the VR environment to enhance their exercise.

The participants were seated on office chairs with wheels, allowing for minor movement, in an electrically 
shielded room during the experiment. The room was divided into two separate sections so there was no visual contact between the participants. EEG, ECG, EDA and breathing were measured from both participants. The experiment consisted of baseline measurement session, meditation activity, and self-reporting.

The experiment was conducted in the DYNECOM environment, and participants wore Oculus Rift VR-glasses for its duration. The experimental procedure took two to three hours for each pair, including the time it took to attach the neurophysiological measuring equipment and breaks in between different conditions. The duration for each pair differed due to technical aspects as well as individual differences in the time it took to fill out the questionnaires. As wearing VR-glasses can cause nausea and the experimental design's duration was rather lengthy, the participants were encouraged to take as many breaks in between subsections of the experiment as they preferred. A majority of the participants ended up taking one or two breaks, during which the VR-glasses were taken off and participants were offered water.

\subsection{Design}

The experiment consisted of eight different conditions, all of which included a baseline measurement, meditation, and a questionnaire about the meditation experience. The baseline measurement was conducted in a VR-room with an "X" on the wall. The participants were in the baseline room until both had been there for two minutes.

After two minutes the participants were transferred within the VR to the meditation environment. The scenarios differed from each other through which parts of the adaptation were active, and whether or not the other person's avatar was active in the environment. Four of the meditation environment scenarios were solo scenarios, where the avatar of the other participant was not active and the other four were dyadic scenarios where the other participant's avatar was active. Both solo and dyadic scenarios included the same four settings: no adaptation, respiration adaptation, EEG adaptation, and respiration adaptation together with EEG adaptation. The meditation scenarios were played in the same order within each pair of participants but in a random order between participant pairs. Each meditation session took six and a half minutes. If the adaptations were active in the scenario, the environment started adapting to the participants' neurophysiological responses after the first 30 seconds. After the meditation period, the participants were transferred within the VR to fill out the self-reports, which they completed using the Oculus Touch VR hand controllers.

\subsection{Participants}

The participants consisted of 42 volunteers (39 female) that were recruited through student e-mail lists. The participant's age ranged between 20 and 50 years, with a mean of 27.09 years $(S D=6.61)$. All participants were given two movie tickets for their participation. The participants were recruited as pairs and therefore all knew their pair before the experiment. The pairs did not involve participants who were in a romantic relationship with each other. All the participants were over 18 years old, right handed, fluent in Finnish and did not report to having any diagnosed neurological or neuropsychological disorders. All participants had completed a minimum of high school education, and 31 of them were part time or full time students at the time of the research. 30 of the participants were in part time or full time employment. One pair of participants wanted to stop the experiment after completing half of the conditions.

The relationships between the participant pairs varied from long ones (23 years) to shorter ones (1 year) with a mean of roughly 8 years. The participants evaluated their relationships to their pairs on a scale from 1 (casual acquaintance) to 9 (best friends). The closeness reported varied between 2 and 9, resulting in a 7.45 average across all participants.

\subsection{Measures}

The measures used in the research were the values in the VR $\log$ files related to the working of the virtual environment and the self-reported data after each condition.

Values for calculated visualizations and the presence of physiological synchrony were logged in the test systems once per second, as defined by the second long epoch measurements in OpenViBE. Frontal asymmetry was saved as value between 0 and 1 , directly pointing to a color on the gradient. When these values from both participants were compared, difference smaller than 0.1 indicated duration when glow- sync effect was shown. For respiration the following data were recorded: aura scale factor, moments when breathing bar waves were sent, and for the synchrony, the seconds when wave collision effect was initialized. However, in the current paper the respiratory indices were not analyzed as EEG was the main physiological measure for empathy, and respiration was included in the study mainly as a natural mechanistic way for the participants to manipulate the biofeedback in the environment, and there was no instruction for the participants to actively 


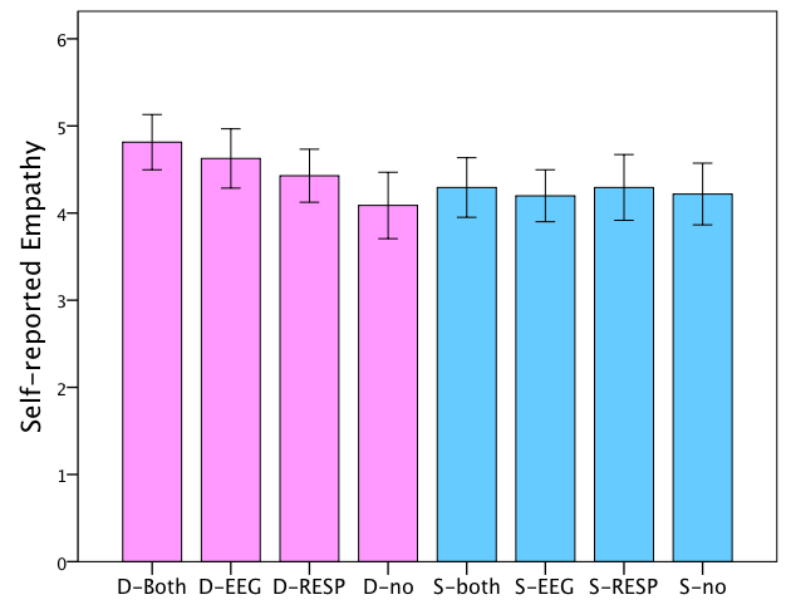

Figure 4. Self-reported empathy in different conditions. $\mathrm{D}=$ dyadic meditation; $\mathrm{S}=$ solo

meditation; both $=$ both, EEG and respiration based adaptations were on; EEG = only EEG based adaptation was on; RESP $=$ only respiration based adaptation was on; no $=$ no adaptation.

pursue synchrony of their respiration rates.

After each condition the participants were asked to rate, on a scale from 1 (not at all) to 7 (extremely) how much they had felt empathy, sympathetic, compassionate, soft-hearted, warm, tender, and moved. The mean of these ratings were used in the analyses as a value for the self-reported empathy, following the work of Batson et al. [43].

\section{Results}

The core findings of the study can be summarized as follows:

1. Evoking empathy is significantly easier when the VR space is shared and compassion meditation is directed at an active avatar

2. Respiration and EEG synchrony feedback together increase self-reported empathy

3. The synchrony biofeedbacks together increase the amount of EEG synchrony

When conducting pairwise analyses, it was observed that there was more self-reported empathy after sessions where the empathy was targeted to an avatar (where the avatar represented the other human participant, thus being a social or dyadic situation) than when it was targeted towards an agent, or a "cold" statue (a non-social or solo situation), $t(1,43)=2.66, p=.01$ (see Figure 4).
In addition, there was more EEG frontal asymmetry indicating approach motivation and empathy in dyadic meditation conditions $(M=.46, S D=.04)$ than in solo conditions $(M=.44, S D=.07), t(1,43)=2.75, p=.01$.

The highest amount of self-reported empathy was evoked after the dyadic meditation sessions, where both the adaptations (EEG and respiration based) were active (Figure 4). The difference in the self-reported empathy between the both adaptations on -condition, and the no adaptations on -condition was statistically significant, $t(1,41)=3.63, p=.001$, so that empathy was reported higher with both of the adaptations than without any adaptations (Figure 4). In addition, reported empathy was higher in the EEG adaptation condition than in the no adaptations condition, $t(1,41)=3.05, p=.004$. Also, in both EEG adaptation conditions, the reported empathy was higher than in the respiration adaptation only condition, $t(1,41)=2.70, p=.01$.

When analyzing the synchronization between the two participant's EEG frontal asymmetries, it was observed that the synchronization was stronger when both the adaptations (EEG and respiration) were on $(M$ $=219.33, S D=59.9)$, than when only the EEG based adaptation was on $(M=178.48, S D=72.43), t(1,41)=$ $2.59, p=.01$.

\section{Discussion}

Our experiment showcased how dyadic biofeedback, based on two persons' respiration and EEG, can augment both self-reported and neurophysiologically measured empathy in virtual environments, when performing an empathy evoking exercise. Furthermore, the results indicate that empathy is more easily evoked in a social than in a solitary meditation condition, that is, when the VR space is shared with another person and the activity is directed at an avatar instead of an agent.

The experimental setup and the environment had some limitations that should be taken into account when interpreting the results and assessing the generalizability of the implications. Mainly, the limitations appeared as difficulties in making the virtual environment operate smoothly. For example, natural chest movements of the avatar, following respiration rhythm, had to be removed due to challenges with the network coding in Unity, and consequently only the more metaphoric respiration waves feedback was shown to the users. The color coding of EEG neurofeedback that was based on the idea of warm and cold colors, could have been counter-intuitive for those participants who interpreted them through a traffic lights metaphor (green $=$ go, red $=$ stop), though the misinterpretations were countered with explicit written instructions that 
clearly stated the meaning of the colors. Still, with biofeedback, intuitiveness is much preferred over mere rational understanding. As is typical with extended VR sessions, some participants experienced some degree of VR sickness despite this element being taken into account beforehand in several ways in the test setup and in the design of the environment itself. However, in hindsight, the avatar to which the participant's focus was mostly directed at, incorporated too many fine details. Given the relatively low resolution of the system, this may have caused flickering that possibly contributed to some participants' reports of discomfort. Another consideration is the gender distribution of the sample where overwhelming majority were females. It possibly skews empathy related results, and consequently it must be taken into consideration when assessing the generalizability of the current results. Additional data has already been collected that balances this difference, but could not be analyzed in time for this paper. From that data set the plan is to additionally analyze the EEG, ECG, EDA and respiration signals and conduct more advanced statistical analysis for a future publication.

In addition to illustrating the strong potential for augmenting compassion meditation in VR and hence supporting the wellness and health benefits associated with meditation practice, these results suggest a broader impact. Dyadic neurofeedback pushes forward the possibilities of biofeedback, providing us with a novel method for feeding back information on low level core social processes such as presence, empathy and emotional connection. This idea can be utilized in a much wider spectrum of affective computing, ranging from virtual negotiation environments to therapy sessions. Indeed, the studies of physiological synchrony has its roots in patient-therapist interactions, and in many recent visions of the future of health technology VR has been highlighted as the new platform for therapy sessions. Biofeedback has potential to many forms of VR therapy, but dyadic synchrony feedback are naturally particularly useful for forms of therapy with a therapist-patient setting. With this spearheading experiment, we hope to encourage a wide range of experimental studies delving into the very nature of dyadic synchrony and feedback, and hope to see innovative prototypes utilizing them in various technological setups. Additionally, DYNECOM utilized respiration and EEG; other biosignals - particularly those that are more easily deployable to mobile consumer grade devices - such as heart rate or skin conductance based measures, should also be examined and their usefulness in this use case mapped out. In continuation of this work, we aim to further delve into these research topics and also assess the potential for a commercial mobile setup and an application.

\section{References}

[1] C. Shaw, D. Gromala, and A. Seay, "The meditation chamber: Enacting autonomic senses," Proc. of ENACTIVE/07, 2007.

[2] D. Gromala, X. Tong, A. Choo, M. Karamnejad, and C. D. Shaw, "The Virtual Meditative Walk: Virtual Reality Therapy for Chronic Pain Management," Proceedings of the 33rd Annual ACM Conference on Human Factors in Computing Systems, pp. 521-524, 2015.

[3] I. Kosunen, M. Salminen, S. Järvelä, A. Ruonala, N. Ravaja, and G. Jacucci, "RelaWorld: Neuroadaptive and Immersive Virtual Reality Meditation System," in Proceedings of the 21st International Conference on Intelligent User Interfaces - IUI '16, (New York, New York, USA), pp. 208-217, ACM Press, 2016.

[4] C. Coelho, J. Tichon, T. Hine, and G. Wallis, "Media presence and inner presence: the sense of presence in virtual reality technologies," From communication to, 2006.

[5] M. J. Schuemie, P. van der Straaten, M. Krijn, and C. A. van der Mast, "Research on Presence in Virtual Reality: A Survey," http://www.liebertpub.com/cpb, jul 2004.

[6] R. B. Welch, T. T. Blackmon, A. Liu, B. A. Mellers, and L. W. Stark, "The Effects of Pictorial Realism, Delay of Visual Feedback, and Observer Interactivity on the Subjective Sense of Presence," http://dx.doi.org/10.1162/pres.1996.5.3.263, 1996.

[7] A. Lutz, H. A. Slagter, J. D. Dunne, and R. J. Davidson, "Attention regulation and monitoring in meditation.," Trends in cognitive sciences, vol. 12, pp. 163-9, apr 2008.

[8] R. Woolfolk, L. Carr-Kaffashan, T. McNulty, and P. Lehrer, "Meditation training as a treatment for insomnia," Behavior Therapy, 1976.

[9] H. Benson, H. P. Klemchuk, and J. R. Graham, "The usefulness of the relaxation response in the therapy of headache," Headache: The Journal of Head and Face Pain, vol. 14, pp. 49-52, apr 1974.

[10] R. Sudsuang, V. Chentanez, and K. Veluvan, "Effect of Buddhist meditation on serum cortisol and total protein levels, blood pressure, pulse rate, lung volume and reaction time," Physiology \& Behavior, 1991.

[11] A. Chiesa and A. Serretti, "A systematic review of neurobiological and clinical features of mindfulness meditations," Psychological Medicine, vol. 40, no. 8, pp. 1239-1252, 2010.

[12] C. Hutcherson, E. Seppala, and J. Gross, "Loving-kindness meditation increases social connectedness.," Emotion, 2008.

[13] S. G. Hofmann, P. Grossman, and D. E. Hinton, "Loving-kindness and compassion meditation: potential for psychological interventions.," Clinical psychology review, vol. 31, pp. 1126-32, nov 2011.

[14] L. Chittaro and A. Vianello, "Computer-supported mindfulness: evaluation of a mobile thought distancing application on naive meditators," International Journal of Human-Computer Studies, 2014. 
[15] C. Carissoli, D. Villani, and G. Riva, "Does a Meditation Protocol Supported by a Mobile Application Help People Reduce Stress? Suggestions from a Controlled Pragmatic Trial," http://www.liebertpub.com/cyber, jan 2015.

[16] A. D. Craig, "How do you feel? Interoception: the sense of the physiological condition of the body.," Nature reviews. Neuroscience, vol. 3, pp. 655-66, aug 2002.

[17] B. Dunn, H. Galton, R. Morgan, D. Evans, C. Oliver, M. Meyer, R. Cusack, A. Lawrence, and T. Dalgleish, "Listening to Your Heart - How Interoception Shapes Emotion Experience and Intuitive Decision Making," Psychological Science, vol. 21, no. 12, pp. 1835-1844, 2010.

[18] J. Cacioppo, L. Tassinary, and G. Berntson, Handbook of psychophysiology. New York, NY: Cambridge University Press, 2007.

[19] J. Waterworth and E. L. Waterworth, "Relaxation Island : A Virtual Tropical Paradise. Interactive Experience," 2004.

[20] Hinterberger and Thilo, "The sensorium: a multimodal neurofeedback environment," Advances in Human-Computer Interaction, vol. 2011, p. 3, 2011.

[21] C. Sas and R. Chopra, "MeditAid: a wearable adaptive neurofeedback-based system for training mindfulness state," Personal and Ubiquitous Computing, vol. 19, no. 7, pp. 1169-1182.

[22] I. Kosunen, A. Ruonala, M. Salminen, S. Järvelä, N. Ravaja, and G. Jacucci, "Neuroadaptive Meditation in the Real World," in Proceedings of the 2017 ACM Workshop on An Application-oriented Approach to BCI out of the laboratory - BCIforReal '17, (New York, New York, USA), pp. 29-33, ACM Press, 2017.

[23] J. E. Escalas and B. B. Stern, "Sympathy and Empathy: Emotional Responses to Advertising Dramas," Journal of Consumer Research, vol. 29, pp. 566-578, mar 2003.

[24] A. J. Hofelich and S. D. Preston, "The meaning in empathy: Distinguishing conceptual encoding from facial mimicry, trait empathy, and attention to emotion," http://dx.doi.org/10.1080/02699931.2011.559192, 2011.

[25] L. M. Oberman, P. Winkielman, and V. S. Ramachandran, "Face to face: Blocking facial mimicry can selectively impair recognition of emotional expressions," http://dx.doi.org/10.1080/17470910701391943, 2007.

[26] J. Coke, C. Batson, and K. McDavis, "Empathic mediation of helping: A two-stage model.," Journal of personality and social, 1978.

[27] L. Aftanas and S. Golocheikine, "Human anterior and frontal midline theta and lower alpha reflect emotionally positive state and internalized attention: high-resolution EEG investigation of meditation," Neuroscience letters, 2001.

[28] W. Klimesch, M. Doppelmayr, and H. Russegger, "Induced alpha band power changes in the human EEG and attention," Neuroscience, 1998.

[29] W. Klimesch, "EEG alpha and theta oscillations reflect cognitive and memory performance: a review and analysis," Brain research reviews, 1999.

[30] T. Field and M. Diego, "Maternal Depression Effects on Infant Frontal Eeg Asymmetry," http://dx.doi.org/10.1080/00207450701769067, 2009.
[31] E. Harmon-Jones, "Clarifying the emotive functions of asymmetrical frontal cortical activity," Psychophysiology, vol. 40, pp. 838-848, nov 2003.

[32] N. Jones, T. Field, M. Davalos, and S. Hart, "Greater right frontal EEG asymmetry and nonempathic behavior are observed in children prenatally exposed to cocaine," http://dx.doi.org/10.1080/00207450490422786, 2009.

[33] C. Marci, J. Ham, E. Moran, and S. Orr, "Physiologic correlates of perceived therapist empathy and social-emotional process during psychotherapy," The Journal of nervous and, 2007.

[34] R. Levenson and A. Ruef, "Empathy: A physiological substrate.," Journal of Personality and Social Psychology, vol. 63, no. 2, p. 234, 1992.

[35] OpenViBE Developers, “Openvibe," 2017.

[36] Unity Technologies, "Unity3d," 2017.

[37] Blender Foundation, "Blender," 2017.

[38] Adobe Systems, "Photoshop cc," 2014.

[39] M. Mori, K. F. MacDorman, and N. Kageki, "The uncanny valley [from the field]," IEEE Robotics Automation Magazine, vol. 19, pp. 98-100, June 2012.

[40] J. J. LaViola, Jr., "A discussion of cybersickness in virtual environments," SIGCHI Bull., vol. 32, pp. 47-56, Jan. 2000.

[41] M. Alger, "Visual Design Methods for Virtual Reality," 2015.

[42] J. Allen, J. Coan, and M. Nazarian, "Issues and assumptions on the road from raw signals to metrics of frontal EEG asymmetry in emotion," Biological psychology, 2004.

[43] C. D. Batson, J. Fultz, and P. A. Schoenrade, "Distress and Empathy: Two Qualitatively Distinct Vicarious Emotions with Different Motivational Consequences," Journal of Personality, vol. 55, pp. 19-39, mar 1987. 